\title{
Spray-Dispersion of Ultra-Small EMT Zeolite Crystals in Thin-Film Composite Membrane for High-Permeability Nanofiltration Process
}

Guodong Kong ${ }^{\text {a, Il }}$, Lili Fan ${ }^{\text {b, Il }}$, Lei Zhao ${ }^{\text {c }}$, Yang Feng ${ }^{\text {a }}$, Xiaolei Cui ${ }^{\text {a }}$, Jia Pang ${ }^{\text {a }}$, Hailing Guo $^{\mathrm{c}}$, Haixiang Sun $^{\mathrm{b}}$, Zixi Kang ${ }^{\mathrm{b}, \mathrm{d}, *}$, Daofeng Sun ${ }^{\mathrm{b}}{ }^{*}$, Svetlana Mintova $^{\mathrm{c}, \mathrm{e}}$

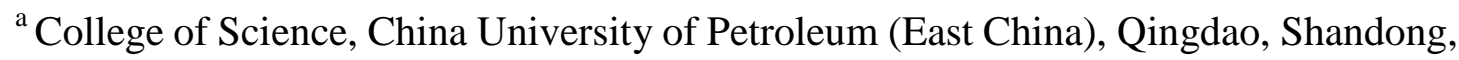
266580, PR China.

${ }^{\mathrm{b}}$ School of Materials Science and Engineering, China University of Petroleum (East China), Qingdao, Shandong, 266580, PR China. kzx@upc.edu.cn (Z. Kang) dfsun@upc.edu.cn (D. Sun)
}

${ }^{c}$ State Key Laboratory of Heavy Oil Processing, Key Laboratory of Catalysis, China National Petroleum Corp. (CNPC), China University of Petroleum (East China), Qingdao, Shandong, 266580, PR China.

${ }^{d}$ State Key Laboratory of Structural Chemistry, Fujian Institute of Research on the Structure of Matter, Chinese Academy of Sciences, Fuzhou, Fujian, 350002, PR China.

e Normandie Universite, ENSICAEN, UNICAEN, CNRS, Laboratoire Catalyse et Spectrochimie (LCS), 14050 Caen, France

II These authors contributed equally to this work. 


\begin{abstract}
Thin-film composite (TFC) membranes show great potential in dye nanofiltration but their performance still required further improvement. In this work, ultra-small EMT zeolite nanocrystals were embedded into the interfacial polymerized polyamide (PA) layer supported on porous nylon via spray dispersion method and used in dye separation. Ultra-small nanosized EMT zeolite crystals with plate-like morphology were synthesized from organic-free precursor suspensions at ambient conditions (30 $\left.{ }^{\circ} \mathrm{C}\right)$. The plate-like EMT nanocrystals were homogeneously dispersed in the thin-film nanocomposite (TFN) membranes, mitigating the formation of agglomerates during the interfacial polymerization. The TFN membrane prepared with $0.05 \mathrm{w} / \mathrm{v} \%$ EMT nanocrystals had a water permeance up to $24.37 \mathrm{~L} \mathrm{~m}^{-2} \mathrm{~h}^{-1}$ bar $^{-1}$, which is nearly 6 times higher than the pristine PA TFC membrane, and maintain the dye rejection (crystal violet) of $99.98 \%$. A new method for preparation of high quality TFN membranes containing zeolite nanocrystals was developed. The potential use of the TFN membranes with the ultra-small zeolite crystals for dye nanofiltration was demonstrated.
\end{abstract}

Keywords: Thin-film nanocomposite membranes; Nanofiltration; Nanosized zeolite; Spray dispersion. 


\section{Introduction}

Wastewater from the textile and printing industry is one of the main cause of current water pollutions.[1-4] Dyes are toxic to aquatic life even at a very low concentration, so the removal of dyes from textile and dyeing wastewater before discharging into the environment is of great importance.[5-8] Technologies including the chemical oxidation, photo-catalysis, coagulation and membrane filtration process have been developed to treat the dye wastewater.[9-13] Membrane separation technology with features as low-cost, energy-saving, and easy installation is attractive for wastewater treatment and water recycling processing. Nanofiltration (NF) has received more attention especially for water purification and pollution control owing to its high permeate flux (compared to reverse osmosis, RO), and excellent rejection rate of divalent and multivalent ionic contaminants compared to ultra-filtration.[14, 15] Both high water flux and dye rejection are essential for the development of nanofiltration membranes. Thin-film composite (TFC) membranes with a high separation performance and stability over a wide range of $\mathrm{pH}$ and temperature, are usually prepared through interfacial polymerization (IP) of a diamine and an acyl chloride on a porous support membrane, which are most frequently used for the NF process.[16] Thin-film nanocomposite (TFN) membranes containing a variety of fillers, such as (silver nanoparticles, metal-organic frameworks (MOFs), covalent organic frameworks (COFs), zeolites, etc.) have been developed to enhance the hydrophilicity, permeability and selectivity of the membranes.[17-20] Deng et al. introduced silver nanoparticles (Ag NPs) with a mean diameter of $15 \mathrm{~nm}$ in the polyamide TFC membrane via covalent bonding using a linking agent (cysteamine).[17] Compared with the original TFC membrane, the water permeance of the TFN membrane modified with Ag NPs increased from 2.41 to $3.36 \mathrm{~L} \mathrm{~m}^{-2} \mathrm{~h}^{-1} \mathrm{bar}^{-1}$, and the retention rate for sodium chloride was only reduced by $<3 \%$. The impermeable fillers can increase membrane permeability through enhancing the surface hydrophilicity, which may also compromise the solute rejection. The introduction of ordered porous nanoparticles may solve this problem. MOFs with uniform pores, which are compatible with polymers, now have been widely studied as a filler of TFC membranes. Bruggen et al. embedded hydrophilic ZIF-8 (Zn-based MOF) NPs into the polyamide (PA) layer of TFC, which doubled the water permeability $\left(14.9 \mathrm{~L} \mathrm{~m}^{-2}\right.$ $\mathrm{h}^{-1}$ bar $^{-1}$ ) without sacrifice of the rejection degree (99.2\%).[21] Moreover, the entire 
organic-organic covalent bonds inside COFs endow them better affinity with organic polymers. Su et al. prepared TFN membranes incorporated with COFs nanoparticles, which showed increased ethanol permeance of $7.98 \mathrm{~L} \mathrm{~m}^{-2} \mathrm{~h}^{-1} \mathrm{bar}^{-1}$ at $0.05 \mathrm{w} / \mathrm{v} \%$ concentration, 46.7\% higher than the pristine TFC membranes.[18] However, the issues such as complex organic ligands, large amount of organic solvents and energy needed by solvothermal synthesis should be concerned for the scale-up preparation and practical application. As another kind of fillers, zeolites with high porosity, regular channel system, and most importantly with high hydrothermal stability have been doped into the TFN membranes. Ghaemi et al. prepared TFN membrane with SAPO-34 zeolite nanoparticles, and the water permeability increased gradually from 2.73 to $8.8 \mathrm{~L} \mathrm{~m}^{-2} \mathrm{~h}^{-1}$ bar $^{-1}$ due to high hydrophilicity and nanochannels created in membrane.[22] However, the poor compatibility between the polymer membranes and the inorganic zeolite crystals resultes in heterogeneous dispersion and non-selective interface voids that degrades the NF performance.[23] The nanosized particles and post-modification of zeolite are two main strategies to mitigate this issue, and the former one is usually preferred due to its simpler operation.[24, 25] EMT zeolite with large pores (12-ring window) and hypercages is more suitable candidate to sieve dyes with higher water permeance compared with small pore (8- or 10-member ring) zeolites, while related researches are rarely reported due to the synthesis difficulty of pure phase and usage of organic structure directing agents (OSDAs).

Herein, the S-EMT zeolite with ultra-small size $(\sim 15 \mathrm{~nm})$ are prepared in mild condition without OSDAs, and well incorporated into PA layers by the spraying. The ultra-small EMT zeolite crystals with uniform pore size and high hydrothermal stability are promising filler for the construction of TFN membranes for efficient nanofiltration.[26] The EMT/PA membrane with improved water permeance and dye rejection rates were prepared. The TFN membranes possess the following advantages: (1) the ultra-small EMT nanocryatls $(\sim 15 \mathrm{~nm})$ can be easily combined with the polymers and avoid the interface voids; (2) the facilely spraying method allows to increase the amount of EMT zeolite nanocrystals without the agglomeration; (3) the EMT zeolite nanocrystals are synthesized at ambient conditions with high yield and without OSDAs, thus reducing the energy and chemicals consumption. 


\section{Experiments}

\subsection{Materials}

All the reagents were used as received. Methylene blue $\left(\mathrm{C}_{16} \mathrm{H}_{18} \mathrm{ClN}_{3} \mathrm{~S} \cdot 3 \mathrm{H}_{2} \mathrm{O}, 98 \%\right)$, crystal violet $\left(\mathrm{C}_{25} \mathrm{H}_{31} \mathrm{~N}_{3}\right.$, 99\%), Congo red $\left(\mathrm{C}_{32} \mathrm{H}_{22} \mathrm{~N}_{6} \mathrm{Na}_{2} \mathrm{O}_{6} \mathrm{~S}_{2}\right.$, $\left.99 \%\right)$ were purchased from Sinopharm Chemical Reagent Co., Ltd., China. Nylon membrane filters purchased from GE Healthcare Life Sciences, were used as the support substrate for TFC and TFN membranes (membrane diameter: $47 \mathrm{~mm}$, pore size: $0.2 \mu \mathrm{m}$ and CAT No.: 7402-004). Piperazine (PIP, $\mathrm{C}_{4} \mathrm{H}_{10} \mathrm{~N}_{2}$, > 99\%) were purchased from Chemical Reagent Co., Ltd., China. Trimesoyl chloride (TMC, > 99.0\%) were purchased from Sigma-Aldrich. Cyclohexane $\left(\mathrm{C}_{6} \mathrm{H}_{12}, 99.5 \%\right)$ were purchased from Tian Jin Fuyu Fine Chemical Co., Ltd. Sodium hydroxide $(\mathrm{NaOH},>99 \%)$ were purchased from Sinopharm Chemical Reagent Co., Ltd., China. $\mathrm{NaAlO}_{2}\left(56.7 \% \mathrm{Al}_{2} \mathrm{O}_{3}, 39.5 \% \mathrm{Na}_{2} \mathrm{O}\right)$ and $\mathrm{Na}_{2} \mathrm{SiO}_{3}\left(29 \% \mathrm{SiO}_{2}, 8 \% \mathrm{Na}_{2} \mathrm{O}, 28.85 \mathrm{~g}\right)$ were purchased from Sigma-Aldrich.

\subsection{Syntheses of EMT zeolites}

The ultra-small EMT zeolite nanocrystals (named S-EMT) were synthesize according to the methodology reported earlier.[25] A colloidal suspension was formed and subjected to crystallization at $303 \mathrm{~K}$ for $36 \mathrm{~h}$. The fully crystalline suspension was separated by a centrifuge and washed with deionized water until $\mathrm{pH}=8$ of the decanting solution was reached. The ultra-small EMT zeolite nanocrystals were freeze-dried prior use for spraying.

The larger sized EMT zeolites (named L-EMT) with the diameter of $100 \mathrm{~nm}$ were synthesized according to the previous study.[27]

\subsection{Preparation of PA TFC membrane}

The nylon substrate was first clamped between an acrylic plastic plate and an acrylic plastic frame. Then, $1.6 \%(\mathrm{w} / \mathrm{v})$ PIP aqueous solution was poured into the frame and allowed to penetrate to the nylon support for $10 \mathrm{~min}$. After soaking for $10 \mathrm{mins}$, the PIP aqueous solution was poured out from the mold, and the excess solution on the surface was removed by a blower. Then, $0.1 \%(\mathrm{w} / \mathrm{v})$ TMC in cyclohexane solution was poured gently into the frame. After the reaction for $1.5 \mathrm{mins}$, the oil phase was discarded, and then washed three times with pure cyclohexane. Finally, the membrane was air-dried for another $1 \mathrm{~min}$, then placed in an oven at $323 \mathrm{~K}$ for $10 \mathrm{mins}$. 


\subsection{Preparation of S-EMT/PA and L-EMT/PA TFN membranes}

The procedure for PA TFC membranes was adapted towards the fabrication of S-EMT/PA membranes including the following additional steps (Scheme 1.). Firstly, S-EMT dispersions with various concentrations were prepared by dissolving 25/50/75 mg of S-EMT zeolite nanocrystals in $100 \mathrm{~mL}$ cyclohexane followed by sonication for 2 hours (Table 1), then added into the sprayer. After the removal of PIP solution from the nylon surface with blower, the S-EMT dispersion $(3.6 \mathrm{~mL})$ was sprayed onto the nylon support. After the complete evaporation of cyclohexane, the spraying step was repeated, then evaporating the solvent again. Then, $0.1 \%(\mathrm{w} / \mathrm{v})$ TMC in cyclohexane solution was poured gently into the frame. After the reaction for $1.5 \mathrm{~min}$, the oil phase was discarded, and the TFN membrane was washed three times with pure cyclohexane. Finally, the membrane was air-dried for another $1 \mathrm{~min}$, and placed at $323 \mathrm{~K}$ for $10 \mathrm{~min}$. The L-EMT/PA membranes were prepared using the L-EMT dispersions $(0.05 \%)$ following the same procedure as described above.

S-EMT/PA-2' membrane was prepared similar to the PA TFC membrane, but the S-EMT was dispersed in PIP aqueous solution with the concentration of $0.05 \%$.

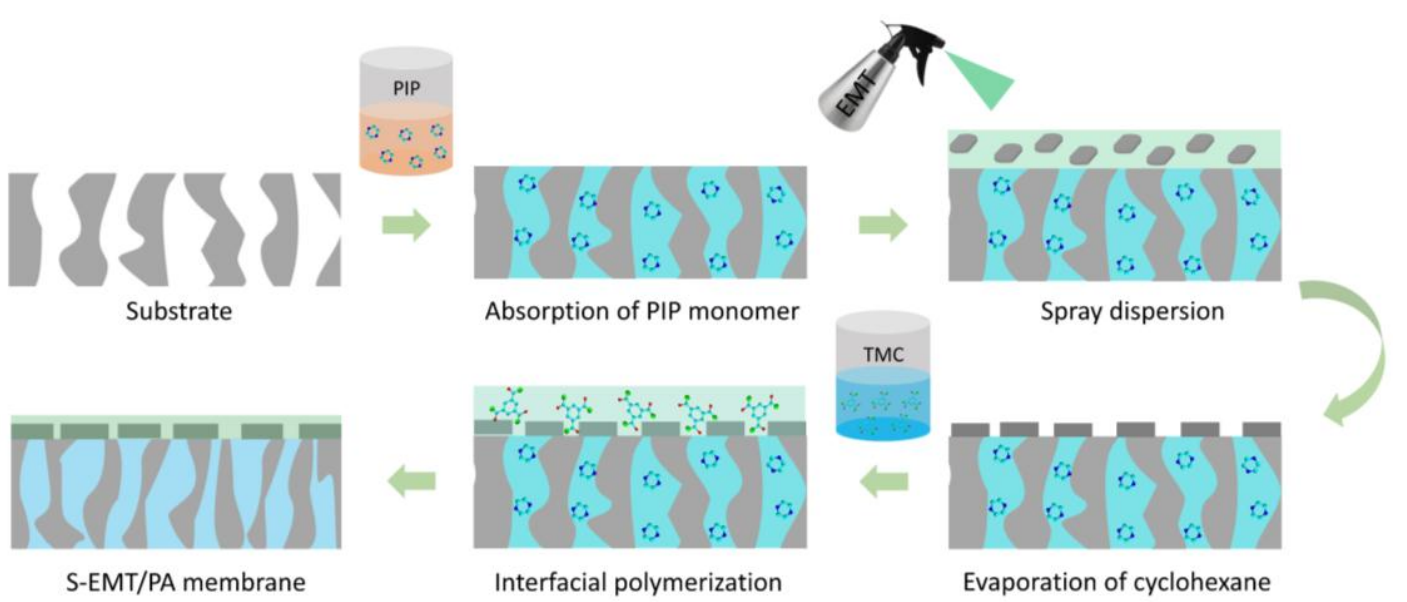

Scheme 1. The process of preparing S-EMT/PA membrane by spraying method.

Table 1. Concentrations of components used for preparing different membranes.

\begin{tabular}{|c|c|c|c|c|}
\hline Membrane & TMC (w/v) & PIP (w/v) & S-EMT(w/v) & L-EMT (w/v) \\
\hline TFC & $0.1 \%$ & $1.6 \%$ & $0 \%$ & $0 \%$ \\
\hline S-EMT/PA-1 & $0.1 \%$ & $1.6 \%$ & $0.025 \%$ & $0 \%$ \\
\hline S-EMT/PA-2 & $0.1 \%$ & $1.6 \%$ & $0.05 \%$ & $0 \%$ \\
\hline
\end{tabular}




\begin{tabular}{|c|c|c|c|c|}
\hline S-EMT/PA-3 & $0.1 \%$ & $1.6 \%$ & $0.075 \%$ & $0 \%$ \\
\hline${ }^{\mathrm{a} S-E M T / P A-2}$ & $0.1 \%$ & $1.6 \%$ & $0.05 \%$ & $0 \%$ \\
\hline L-EMT/PA & $0.1 \%$ & $1.6 \%$ & $0 \%$ & $0.05 \%$ \\
\hline
\end{tabular}

a S-EMT was dispersed in the PIP aqueous solution.

\subsection{Characterization}

X-ray diffraction (XRD) measurements were carried out at room temperature using Ultima powder X-ray diffraction in the range of $5-65^{\circ} 2 \theta$ betweenwith $\mathrm{Cu} \mathrm{K}_{\text {alpha }}$ radiation. The size of the EMT nanocrystals in suspensions was determined by the Dynamic Light Scattering (Brookhaven BI-200SM) at $25{ }^{\circ} \mathrm{C}$. The Brunauer-Emmett-Teller (BET) surface areas of the samples were calculated from the $\mathrm{N}_{2}$ adsorption-desorption isotherms at $77 \mathrm{~K}$ collected with Micromeritics ASAP 2020. The thermal stability of EMT zeolites is determined by thermogravimetric analysis (NETZSCH SAT449 F5) under air atmosphere. The EMT ( $\mathrm{KBr}$ tablets) were characterized by Fourier-transform infrared spectroscopy (FTIR) using a Bruker Tensor 37, German. The zeolite nanocrystals and membranes were characterized using a scanning electron microscope (SEM, HITACHI, S4800). TEM images were taken on Transmission Electron Microscope (FEI Tecnai G20) at an acceleration voltage of $200 \mathrm{kV}$.

The residual concentration of organic dyes in the solution was measured using a UV-visible spectrophotometer (UV-2450, SHIMADZU, Japan) at wavelengths of 750 and $400 \mathrm{~nm}$. The hydrophilicity of membranes was investigated using a droplet contact angle JC2000D meter. Zeta potential values of EMT zeolite crystals were tested by nanoparticle analyzer (SZ-100-Z, HORIBA, Japan). To investigate the surface features of the membranes, a HIMADZU SMP-9700 instrument was used. Dynamic Light Scattering (DLS) was used to confirm the particle size distribution of EMT zeolite crystals with Brookhaven BI-200SM at $25{ }^{\circ} \mathrm{C}$. Attenuated total reflectance Fourier transform infrared spectroscopy (ATR-FTIR, Nicolet-58S) was used to analyze the functional groups on the membrane surfaces.

\subsection{Nanofiltration performance of membranes}

The membrane performance was evaluated using a dead-end filtration device with an effective area of $7.065 \mathrm{~cm}^{2}$. The trans-membrane pressure of the system was 
maintained at $0.1 \mathrm{MPa}$ during the filtration process. To test the retention performance of the membranes, the methylene blue, crystal violet and Congo red with a constant concentration $(10 \mathrm{ppm})$ were used as feed solutions. The average values of three measurements were taken and the standard deviation was determined.

The water passing through the filter device was weighed by an electronic balance and the water permeance $J\left(\mathrm{~L} \mathrm{~m}^{-2} \mathrm{~h}^{-1} \mathrm{MPa}^{-1}\right)$ was calculated by the following equation:

$$
I=\frac{V}{A \times \Delta t \times \Delta P}
$$

Where $V(\mathrm{~L})$ is the volume of the permeated water, $A\left(\mathrm{~m}^{2}\right)$ is the membrane effective area, $\Delta t(\mathrm{~h})$ is the operation time, and $\Delta P(\mathrm{MPa})$ is the operating pressure. The rejection rates was calculated using the following equation:

$$
\mathrm{R}=\left(1-\frac{c_{P}}{c_{f}}\right) \times 100 \%
$$

where $C_{f}$ and $C_{p}$ are the feed and permeate concentrations, respectively.

\subsection{Stability test of membranes}

Long-term stability test of the membrane was carried out in a cross-flow unit with an effective membrane surface of $0.785 \mathrm{~cm}^{2}$. Before the test, the membrane was compacted in the filtration unit with distilled water under pressure of 0.5 bar to reach equilibrium flux for at least $2 \mathrm{~h}$. The system was then operated with $1000 \mathrm{~mL}$ (50 ppm) Congo red, methylene blue, and crystal violet under 3 bar at $25{ }^{\circ} \mathrm{C}$ for 50 hours.

\section{Results and discussion}

\subsection{Characterization of EMT nanosized zeolite crystals}

The FTIR spectra of as-synthesized EMT (S-EMT and L-EMT) zeolite samples are presented in Fig. 1a. Two samples have similar major absorption peaks at wavenumbers of $1645 \mathrm{~cm}^{-1}, 990 \mathrm{~cm}^{-1}, 740 \mathrm{~cm}^{-1}$ and $663 \mathrm{~cm}^{-1}$ assigning to the O-H (stretching vibration), T-O-T (asymmetric stretch) and T-O-T (symmetric stretch), respectively. [28] Both EMT zeolite samples possess identical XRD patterns with the simulated pattern for the EMT framework type framework structure (Fig. 1b). Due to the random stacking, the three characteristic peaks of the EMT zeolite in the region $5-7^{\circ} 2 \theta$ are overlapped, and the relative broadening of the peaks demonstrated the small particles in the sample S-EMT. The size and morphology of the S-EMT crystals can be seen in Fig. 2a, a few hundred nanometers in size agglomerates are clearly observed. The particle size of L-EMT zeolite sample is about $100 \mathrm{~nm}$ in diameter, which is bigger than the crystals of S-EMT sample $(15 \mathrm{~nm})$. The S-EMT crystals with 
a hexagonal plate-like morphology are shown in the TEM images (Fig. 2c). The particle size distribution deduced by DLS for both samples S-EMT and L-EMT is presented in Fig. 3, the DLS results are consistent with the SEM and TEM observations. Given the important role of the surface charge (exclusion) of the EMT nanocrystals in the nanofiltration, the zeta potential measurements in the suspensions containing the S-EMT and L-EMT crystals were performed. The results reveal that the zeta potential values of S-EMT and L-EMT are $-23.6 \mathrm{mv}$ and $-33.9 \mathrm{mv}$ at $\mathrm{pH}=6.8$ (Fig. 4). More zeta potential values at varied $\mathrm{pH}$ can be found in Fig. S1. Since appropriate pore size is very important for porous fillers, the pore size distribution of S-EMT and L-EMT were calculated based on the $\mathrm{N}_{2}$ adsorption isotherms at $77 \mathrm{~K}$. As shown in Fig. S2, both of the two porous materials have similar pore size distribution, which are $0.78 \mathrm{~nm}(\mathrm{~S}-\mathrm{EMT})$ and $0.74 \mathrm{~nm}$ (L-EMT), respectively, indicating that the zeolites are suitable fillers in TFN membrane for separating dyes. The ultra-small size and suitable pore size offer S-EMT great advantages as inorganic fillers for the construction of TFN membranes.
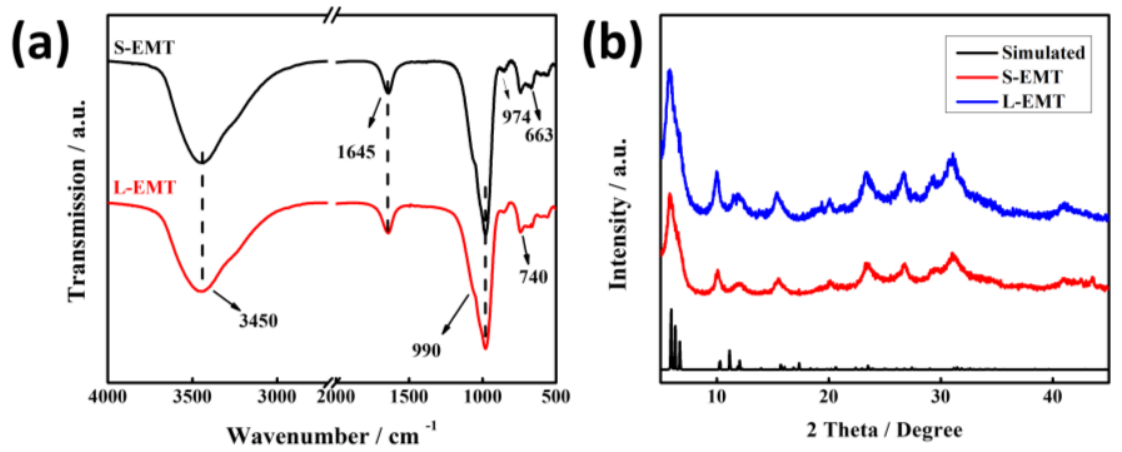

Fig. 1 (a) FTIR spectra and (b) PXRD patterns of S-EMT and L-EMT zeolite samples.
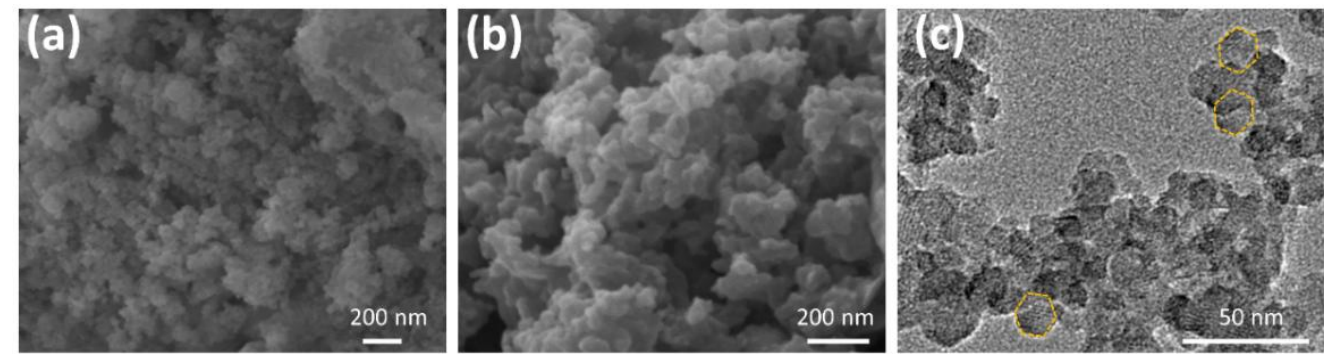

Fig. 2 SEM pictures of (a) S-EMT and (b) L-EMT zeolite samples, and TEM picture of (c) S-EMT. 
(a)

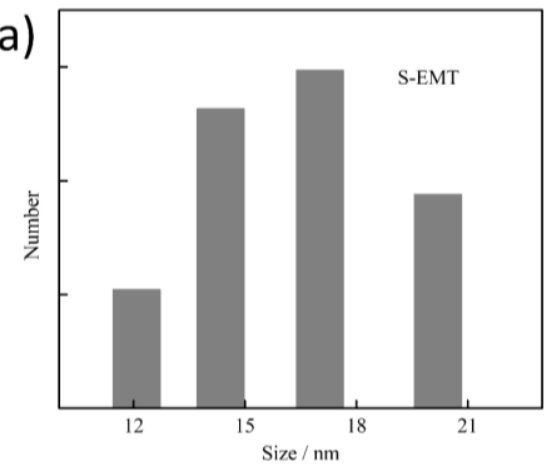

(b)

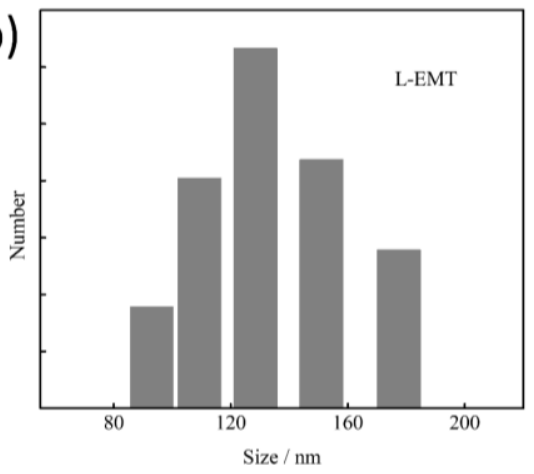

Fig. 3 Particle size distribution of (a) S-EMT and (b) L-EMT zeolites determined by DLS.
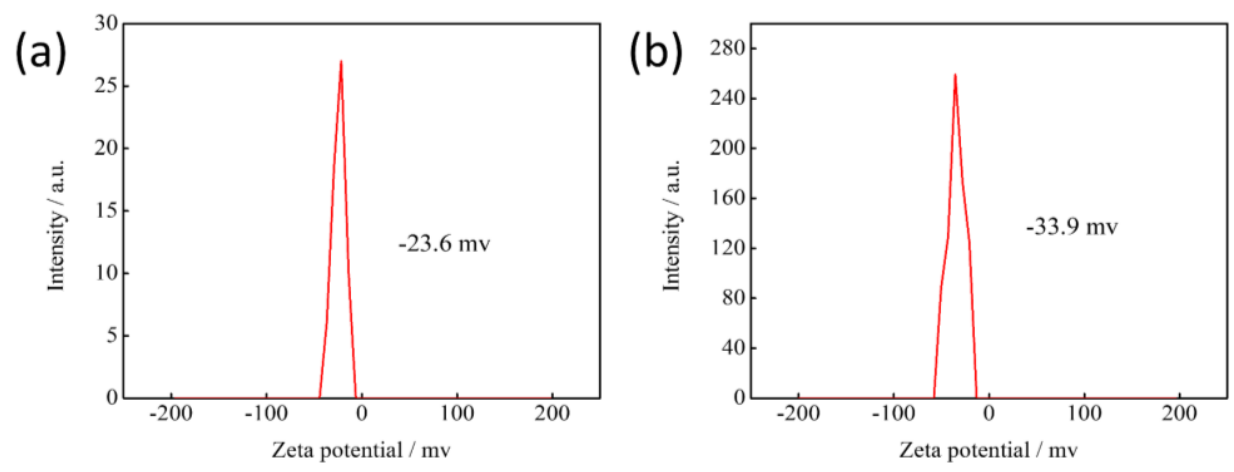

Fig. 4 Zeta potential curves of (a) S-EMT, (b) L-EMT zeolite samples.

\subsection{Characterization of PA TFC and EMT/PA TFN membranes}

The ATR-FTIR spectra of PA TFC and EMT/PA TFN membranes and the nylon support are shown in Fig. S5. The intensity of two peaks at $3300 \mathrm{~cm}^{-1}$ and $1640 \mathrm{~cm}^{-1}$ are found to be slightly enhanced for the PA TFC and EMT/PA TFN membranes, which is associated with the vibrations of amide groups, $\mathrm{C}-\mathrm{N}$ and $\mathrm{N}-\mathrm{H}$, confirming the formation of PA on the nylon substrate. Due to the low amount of EMT zeolite nanocrystals as a filler $(<0.075 \mathrm{wt} \%)$ in the polyamide layer, the IR spectrum of the EMT/PA membrane does not contain any vibration peaks characteristic of the zeolite (stretching Si-OH vibration at $970 \mathrm{~cm}^{-1}$ and asymmetric $\mathrm{Si}-\mathrm{O}-\mathrm{Si}$ vibration at $740 \mathrm{~cm}^{-1}$ ). The XRD patterns do not exhibit the Bragg peaks of EMT zeolite, and only three broad peaks at $17.72,22.58$ and $25.99^{\circ} 2$ Theta belonging to the nylon substrate are present (Fig. 5). 


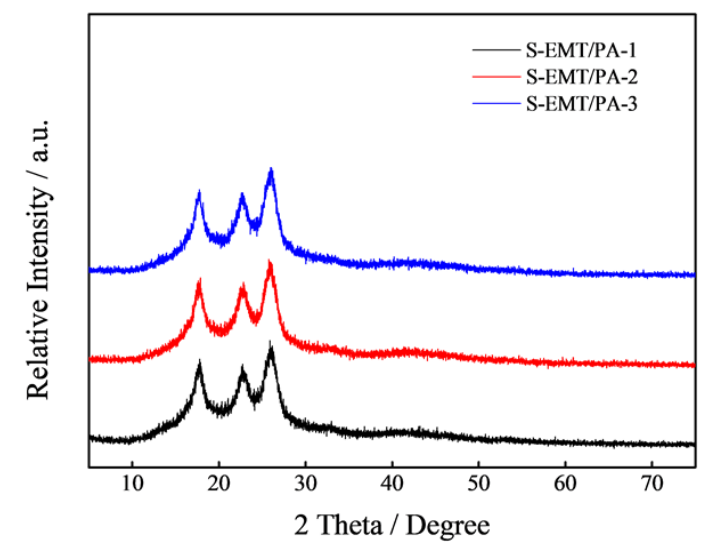

Fig. 5 XRD patterns of S-EMT/PA-1, S-EMT/PA-2 and S-EMT/PA-3 membranes.

(a)
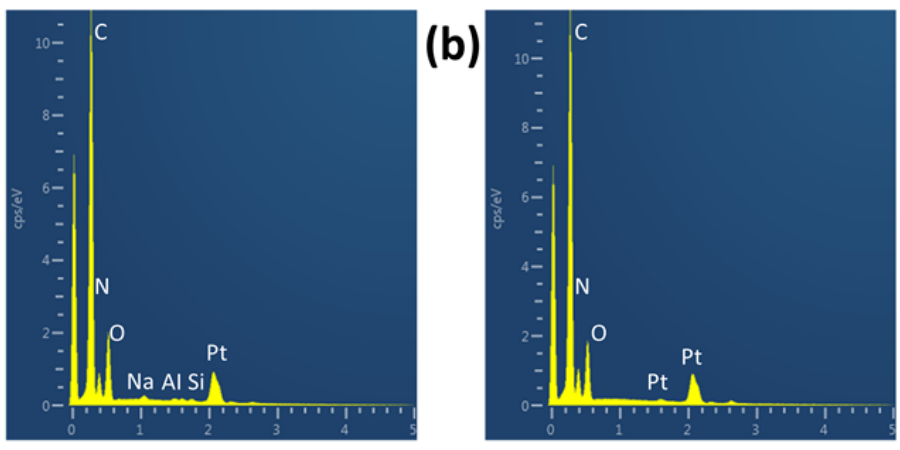

(c)

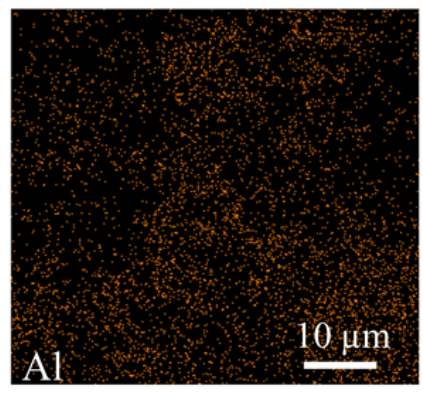

(d)

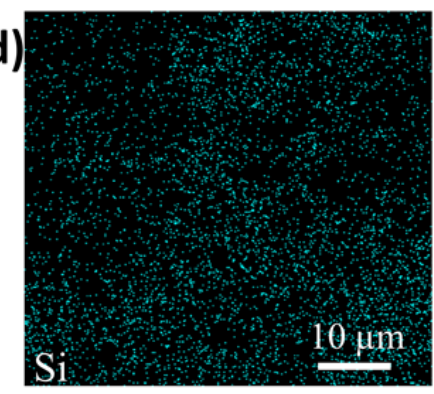

Fig. 6 EDX spectra of (a) S-EMT/PA-2 membrane and (b) PA TFC membrane; EDX mapping of (c) $\mathrm{Al}$ and (d) Si for the S-EMT/PA-2 membrane.

The membranes (PA TFC membrane and S-EMT/PA-2 membrane) were further analyzed using energy dispersive $\mathrm{X}$ - ray spectroscopy (EDX) to confirm whether zeolite crystals are present. The EDX spectrum of the S-EMT/PA-2 membrane contains the $\mathrm{Al}$ peak at $\sim 1.49 \mathrm{keV}$ and $\mathrm{Si}$ peak at $\sim 1.74 \mathrm{keV}$, confirming the presence of the EMT zeolite; these peaks are absent in the spectrum of pure PA TFC membrane 
(Fig. 6). Furthermore, the $\mathrm{O}$ peak at $\sim 0.53 \mathrm{keV}$ is stronger in the S-EMT/PA-2 membrane than in the PA TFC membrane owing to the presence of zeolite crystals. [20] The surface features of the PA TFC and EMT/PA TFN membranes were characterized by SEM and AFM. As shown in Fig. 7, the membrane surface of pristine PA TFC membrane (Fig. 7a) shows a typical "nodular" structure, which is the common observed for PA formed via interfacial polymerization.[29] The hydrophilic pores of the nylon substrate make the PIP aqueous solution in the pores appearing concave. The water soluble monomer diffusing slowly is restricted by the hydrophilic support, and eventually formed a relatively smooth nodular structure.[30] However, the S-EMT/PA membranes' surface show a "ridge and valley" morphology explained with the presence of EMT nanosized zeolite crystals incorporated in the PA layer. The hydrophilic zeolite nanocrystals may adsorb a certain amount of PIP monomer, so that the reaction of TMC and PIP monomer is not limited by the pore size of the substrate. PIP monomer absorbed by zeolites may react with TMC to form nucleus of polyamide, which further evolves into polyamide tufts. The different growth orientations of tufts and lateral diffusion of PIP may lead to a "ridge and valley" structure.[21] As shown by SEM, the S-EMT/PA membranes have gradually increased roughness which is caused by the increased incorporation of zeolite nanocrystals from S-EMT/PA-1 to S-EMT/PA-3. Compared with the S-EMT/PA, the L-EMT/PA membrane exhibits rougher surface due to the presence of individual L-EMT crystals not embedded in the polymer.
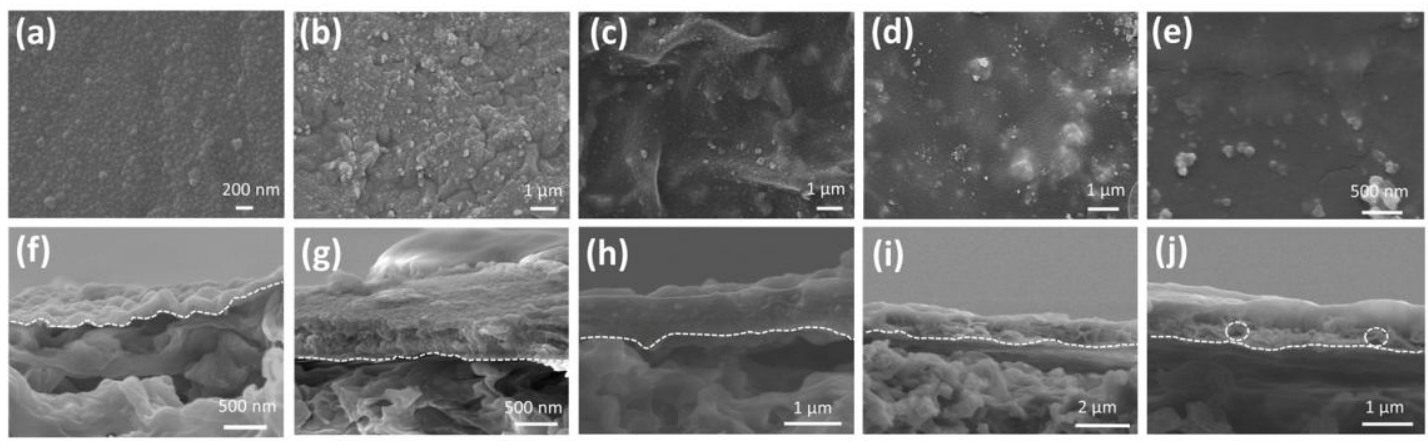

Fig. 7 The top-view and cross-section SEM images of (a and f) PA TFC, (b and g) S-EMT/PA-1, (c and h) S-EMT/PA-2, (d and i) S-EMT/PA-3 and (e and j) L-EMT/PA membrane. 
The cross-section SEM images reveal some voids and poorly attached interfaces of the L-EMT and the PA active layer (Fig. 7e, j). While the S-EMT/PA-2 membrane exhibits smooth and clean surface without voids (Fig. 7c, h). Small-sized zeolites are easier to combine with polymers and mitigate the generation of interface defects.[24] More cross-section images were collected (Fig. S6) of the membranes with a larger magnification to prove this point. From the Fig. S6, it can be seen that the S-EMT/PA-2 membrane has a smooth and uniform cross-section surface. However, the cross-section view of L-EMT/PA membrane is relatively rough, and it can be seen that there are some defects between the L-EMT and PA selective layer (white cycles). As shown in Fig. 7, PA membrane has a thickness of $\sim 108 \mathrm{~nm}$, while the thickness increases from $\sim 400 \mathrm{~nm}$ (S-EMT/PA-1) to $\sim 850 \mathrm{~nm}$ (S-EMT/PA-3). This increment of membrane thickness is due to the addition of zeolites during the interfacial polymerization. Although the thickness of the membrane gradually increases, a higher water flux and rejection rate are still ensured due to the porosity and hydrophilicity of the S-EMT in the PA layer. The EDS mapping shows the homogeneous distribution of $\mathrm{Al}$ and $\mathrm{Si}$ in the EMT/PA membranes (Fig. $6 \mathrm{c}$ and d), demonstrating the good distribution of the EMT zeolite nanocrystals in the TFN membranes. To further illustrate the advantages of the spray method, the membrane that was only sprayed (no interfacial polymerization) was characterized by SEM and compared with the membrane prepared by traditional methods (adding S-EMT into the water solution of PIP). As shown in Fig. S7 a-c, S-EMT can be evenly distributed on the surface of the nylon substrate after spraying, which is convenient to combine with the PA layer during the subsequent interfacial polymerization. For traditional dispersion methods, S-EMT zeolites agglomerate into particles with different sizes on the surface of the substrate. This phenomena indicates that the spraying method is beneficial to the dispersion of S-EMT in the PA layer. The surface morphology (Fig. 8) and roughness (Table 2) of the membranes were also investigated by AFM. The average roughness of the S-EMT/PA membranes increased slightly compared with that of the pristine PA TFC membrane, and became rougher with increasing the amount of zeolite nanocrystals added (Table 2). The roughness of the membranes loaded with the same amount of S-EMT and L-EMT zeolites $(0.05 \%)$ have a roughness $\left(R_{a}\right)$ of $263.4 \mathrm{~nm}$ 
and $355.19 \mathrm{~nm}$, respectively. These resulst are consistent with the observations made by SEM.
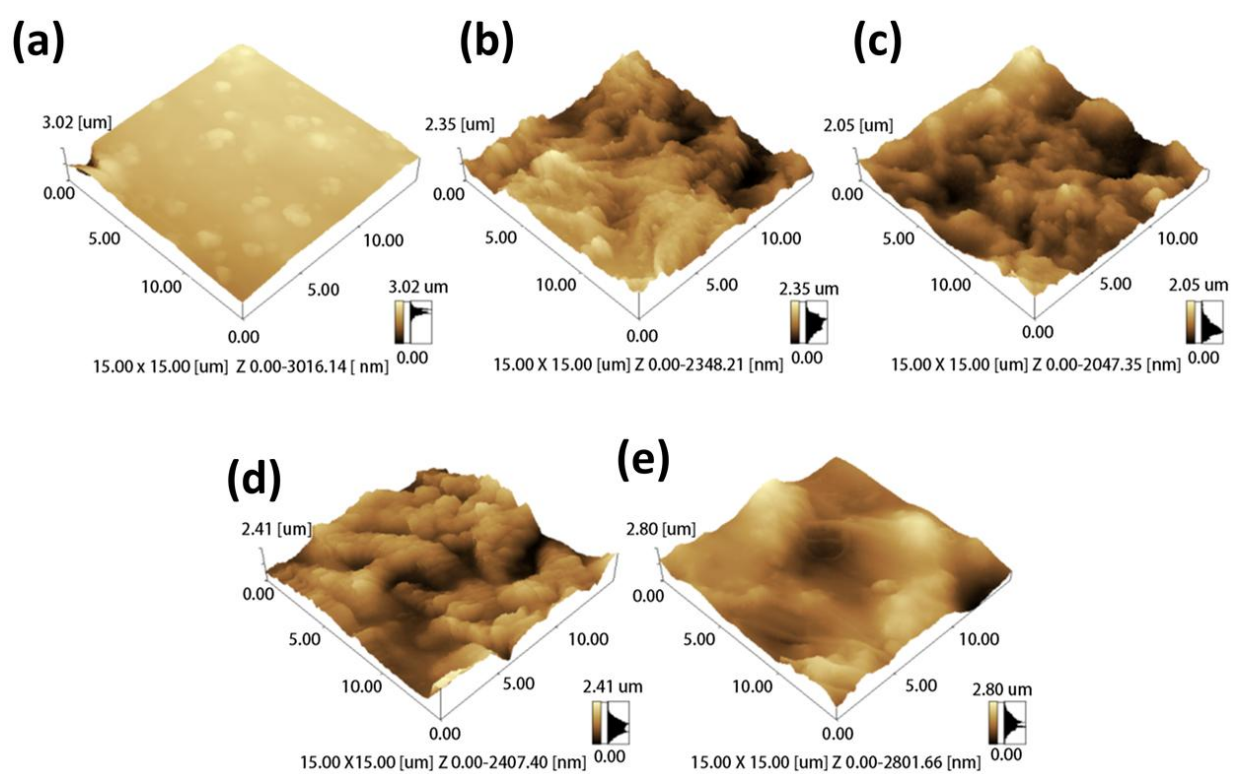

(e)

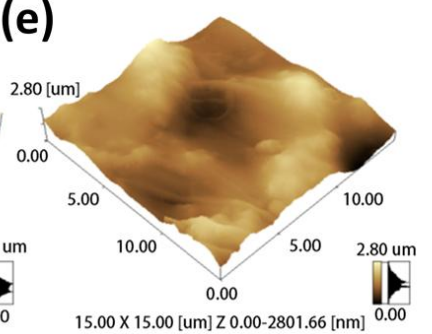

Fig. 8 AFM images of (a) PA TFC, (b) S-EMT/PA-1, (c) S-EMT/PA-2, (d) S-EMT/PA-3 and (e) L-EMT/PA membranes.

Table 2. Roughness (Ra) of membranes measured by AFM.

\begin{tabular}{|l|l|l|}
\hline Membrane & EMT (w/v) & Ra $(\mathbf{n m})$ \\
\hline TFC & $0 \%$ & 84.35 \\
\hline S-EMT/PA-1 & $0.025 \%$ & 258.3 \\
\hline S-EMT/PA-1 & $0.05 \%$ & 263.4 \\
\hline S-EMT/PA-3 & $0.075 \%$ & 311.0 \\
\hline L-EMT/PA & $0.05 \%$ & 355.2 \\
\hline
\end{tabular}




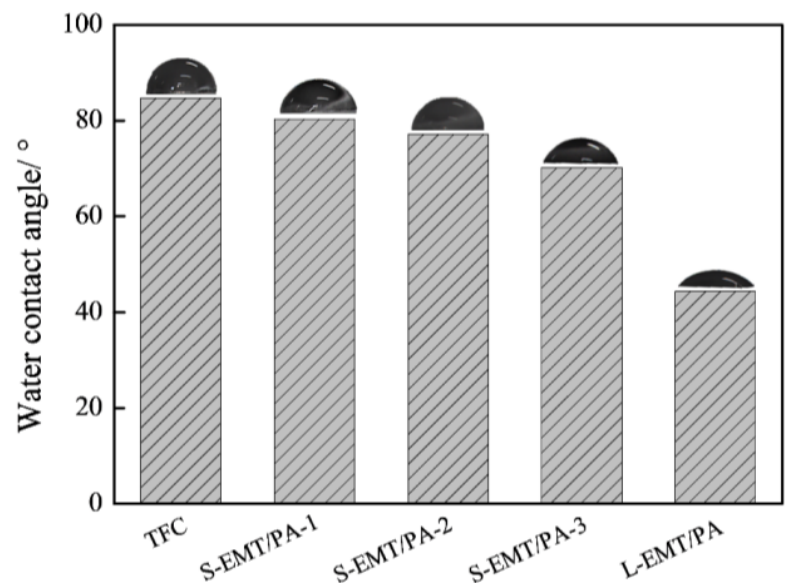

Fig. 9 Water contact angle (CA) measurements of PA TFC and S-EMT/PA-1, -2, -3 and L-EMT/PA-1 membranes.

The water contact angle, measurements were performed to evaluate the hydrophilicity of the membranes that has a great effect on the water flux. The contact angle measurement of S-EMT/PA-1 membrane reduces from $84.8^{\circ}$ to $80.4^{\circ}$ in comparison to the parent PA TFC membrane (Fig. 9). This can be attributed to the presence of S-EMT zeolite with polar groups $(-\mathrm{OH},-\mathrm{COOH})$ on its surface, which improves the hydrophilicity of the TFN membranes.[20] Moreover, water contact angle decreased from S-EMT/PA-1 to S-EMT/PA-3 as increasing the amount of highly hydrophilic S-EMT zeolite nanocrystals. Based on the Wanzel equation, the wetting properties of a solid substance should be directly proportional to the roughness of the surface wetted.[31] It can be explained that the roughness of the membranes surface increases with the amount of EMT nanocrystals added. As more hydrophilic S-EMT zeolite nanocrystals are added from S-EMT/PA-1 to S-EMT/PA-3, the water contact angle gradually decreased. In addition, the L-EMT/PA membrane has the lower water contact angle compared with S-EMT/PA-3. The increase in hydrophilicity is not only due to the increase of roughness, but also related to the addition of hydrophilic L-EMT. Compared with S-EMT, L-EMT with larger size is easier to be exposed on the surface of the membrane (Fig. S8), which further improves the hydrophilicity of the membrane.

\subsection{Nanofiltration performance of EMT/PA TFN membranes}

The nanofiltration properties of PA TFC and EMT/PA TFN membranes were evaluated using the dye of crystal violet feed solution $(10 \mathrm{ppm})$, and the results are summarized in Fig. 10a The S-EMT/PA-2 membrane shows the enhanced water permeance from $3.61 \pm 0.2$ to $24.37 \pm 1.7 \mathrm{~L} \mathrm{~m}^{-2} \mathrm{~h}^{-1}$ bar $^{-1}$ by adding EMT nanocrystals 
without losing the retention $(99.98 \pm 2.6 \%)$. The reason for this performance is that appropriate pore size of the hydrophilic EMT zeolite $(0.78 \mathrm{~nm})$ affords fast diffusion for water molecules and good sieving effect for dyes; For comparison, the membrane with the bigger zeolite crystals (sample L-EMT/PA) prepared at identical conditions as S-EMT/PA-2 membrane was also tested. The non-selective voids formed between the large EMT zeolite nanocrystals and the polymers resulted in lower rejection rate $(61.57 \pm 1.9 \%)$ and higher water permeance $\left(42.57 \pm 3.0 \mathrm{~L} \mathrm{~m}^{-2} \mathrm{~h}^{-1} \mathrm{bar}^{-1}\right)$. The non-selective voids formed between the large EMT zeolite nanocrystals offers more passageways for water and dye molecules. Which indicates that the ultra-small EMT nanocrystals alleviate the compatibility problem between the inorganic filler and the polymer greatly, thus reducing the generation of non-selective voids. Moreover, the nanofiltration performance of S-EMT/PA-2' membrane prepared by traditional methods (Fig. S9) was also tested to illustrate the advantages of the spray method. Fig. S9 shows that the water permeance of S-EMT/PA-2 prepared by spaying is $24.37 \mathrm{~L}$ $\mathrm{m}^{-2} \mathrm{~h}^{-1} \mathrm{bar}^{-1}$, which is much higher than that of S-EMT/PA-2' (12.05 $\left.\mathrm{L} \mathrm{m}^{-2} \mathrm{~h}^{-1} \mathrm{bar}^{-1}\right)$. This result is because of (1) lower loading ratio and S-EMT and (2) the agglomeration of S-EMT caused by the traditional method.

(a)

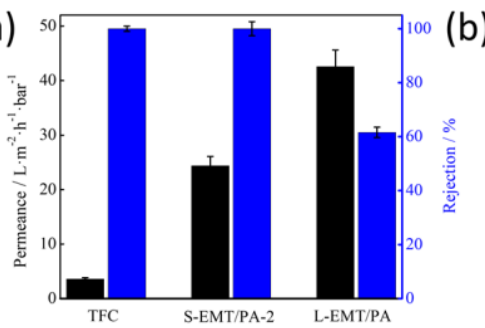

(b)

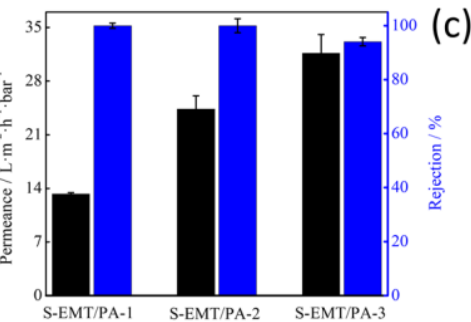

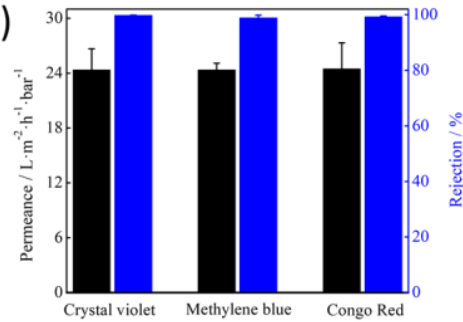

Fig. 10 Water permeance and rejection rate of membranes illustrating (a) the effect of the EMT zeolite crystals with different particle sizes, (b) the effect of the EMT loding amount, and (c) the effect of the dye molecules used.

Table 3. The structural properties of dye molecules and EMT zeolite used in this study.

\begin{tabular}{|l|l|l|l|l|}
\hline & Crystal violet & Methylene blue & Congo Red & EMT \\
\hline & & & \\
& &
\end{tabular}




\begin{tabular}{|l|l|l|l|l|}
\cline { 2 - 5 } $\begin{array}{l}\text { Chemical } \\
\text { structure }\end{array}$ & & & & \\
\hline $\begin{array}{l}\text { Molecular } \\
\text { form }\end{array}$ & $\mathrm{C}_{25} \mathrm{H}_{31} \mathrm{~N}_{3}$ & $\mathrm{C}_{16} \mathrm{H}_{18} \mathrm{ClN}_{3} \mathrm{~S}$ & $\mathrm{C}_{32} \mathrm{H}_{22} \mathrm{~N}_{6} \mathrm{Na}_{2} \mathrm{O}_{6} \mathrm{~S}_{2}$ & \\
\hline Size & $13.05 \times 13.05 \AA$ & $13.17 \AA \times 5.27 \AA$ & $25.60 \AA \times 7.30 \AA$ & $7.8 \AA$ \\
\hline Mol. wt. & $373.53 \mathrm{~g} \cdot \mathrm{mol}^{-1}$ & $319.85 \mathrm{~g} \cdot \mathrm{mol}^{-1}$ & $696.68 \mathrm{~g} \cdot \mathrm{mol}^{-1}$ & - \\
\hline Charge & Positive & Positive & Negative & Negative \\
\hline
\end{tabular}

The effect of EMT zeolite loading $(0.025 \%$ to $0.075 \% \mathrm{w} / \mathrm{v})$ on the NF performance is presented in Fig. 10b. With increasing the concentration of the S-EMT dispersions, the water permeance increases from $13.27 \pm 0.16$ to $31.65 \pm 2.4 \mathrm{~L} \mathrm{~m}^{-2} \mathrm{~h}^{-1} \mathrm{bar}^{-1}$. The increased amount of the used EMT nanosized zeolite crystals resulted in improved hydrophilic membranes thereby enhancing the membrane permeance. Both the S-EMT/PA-1 (99.99 $\pm 0.1 \%)$ and S-EMT/PA-2 (99.98 $\pm 0.26 \%)$ possessed rejection rates higher than for the S-EMT/PA-3 membrane $(94.07 \pm 0.15 \%)$. Although the hydrophilic EMT nanoflakes can enhance the water permeability, there is an optimum zeolite concentration that brings positive changes for the membrane performance. Beyond this optimal level, the permeability may drop due to the pore blocking and aggregation of the excessive zeolite nanocrystals.

In the nanofiltration, both the electrostatic repulsion and molecular sieving have to be taken into account.[32-34] Three different dye molecules with various charges and sizes (methylene blue, crystal violet and Congo red) are used to evaluate the S-EMT/PA-2 membrane (Fig. 10c, Table 3). No matter the charge of the dyes, i.e. the positively-charged dyes (crystal violet and methylene blue) or the negatively-charged dye (Congo red), the retention rate of the S-EMT/PA-2 membrane maintained at a high level (> 98\%). On the one hand, although positively charged dyes have electrostatic attraction with negatively EMT, the rejection rate can still be stable, thanks to the appropriate pore size and good compatibility of the S-EMT zeolites with the polymer. On the other hand, for the negatively charged dye molecules (Congo red), both molecular sieving and electrostatic repulsion ensure a stable retention rate. The S-EMT/PA-2 membrane was also applied to separate ion salt solutions ( $\mathrm{NaCI}, \mathrm{Na}_{2} \mathrm{SO}_{4}$ and $\mathrm{MgCl}_{2}$ ) by nanofiltration. As shown in Fig. S10, the rejection rates of the $\mathrm{Na}_{2} \mathrm{SO}_{4}$, $\mathrm{MgCl}_{2}$ and $\mathrm{NaCl}$ are $50.82 \%, 38.04 \%$ and $28.44 \%$, respectively. The salt ions 
rejection mechanism can be explained by the Donnan exclusion theory and physical sieving effect. On the one hand, the negatively charged S-EMT/PA-2 membrane tends to repel negatively charged ions $\left(\mathrm{SO}_{4}{ }^{2-}\right)$, thus realizing a higher rejection for $\mathrm{Na}_{2} \mathrm{SO}_{4}$ compared with $\mathrm{NaCl}$. On the other hand, the rejection rate of $\mathrm{MgCl}_{2}(38.04 \%)$ is higher than that of $\mathrm{NaCl}(28.44 \%)$ due to the larger hydrated radius value of $\mathrm{Mg}^{2+}$ $(0.43 \mathrm{~nm})$ than $\mathrm{Na}^{+}(0.36 \mathrm{~nm})$. However, the EMT based composite membrane shows low salt retention properties due to the large pore size $(0.78 \mathrm{~nm})$ and hypercages $(1.4$ $\mathrm{nm})$ of EMT zeolites. The performance stability of the membrane is essential in practical applications. The flux under different pressures was tested to confirm the good structural stability. As shown in Fig. 11a, the water flux of S-EMT/PA-2 membrane increases linearly with pressure in the range of 2.0-8.0 bar. The results indicate that the membrane exhibits high mechanical property to maintain the NF performance at high operating pressures. To investigate the durability of the S-EMT/PA-2 membrane, long-term tests were conducted at the operating pressure of 3 bar for $50 \mathrm{~h}$ with $50 \mathrm{ppm}$ Congo red aqueous solution. Fig. 11b shows that the permeance has no significant reduction maintaining at a high level $\left(24.37 \mathrm{~L} \mathrm{~m}^{-2} \mathrm{~h}^{-1}\right.$ bar $^{-1}$ ) during the nanofiltration test. Also, the retention rate is higher than $95 \%$ through the whole process, confirming the stability of the S-EMT/PA-2 membrane.

(a)

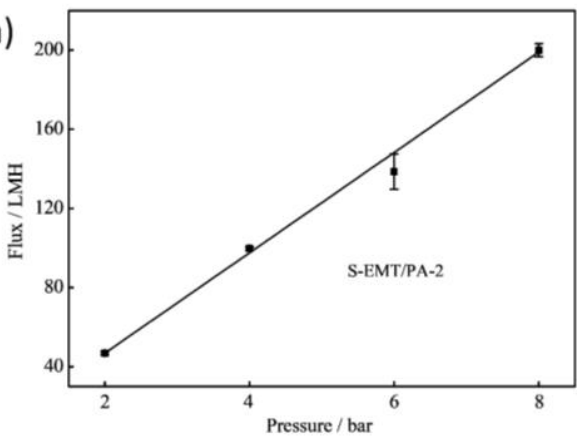

(b)

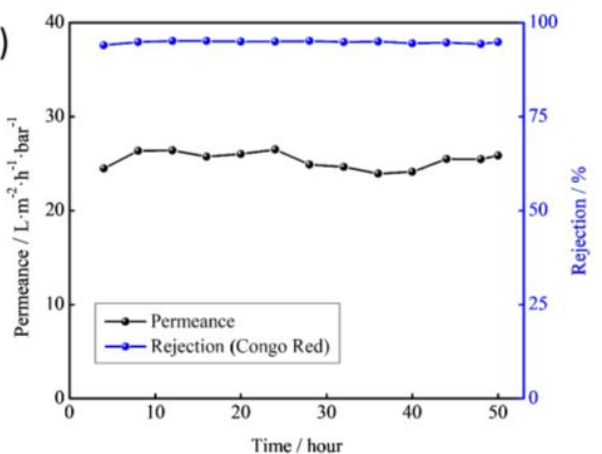

Fig. 11 (a) Water flux of the S-EMT/PA-2 membrane under different pressures. (b) Stability test of the S-EMT/PA-2 membrane at operating pressure of 3 bar and dye (Congo red) concentration of $50 \mathrm{ppm}$ at room temperature. 


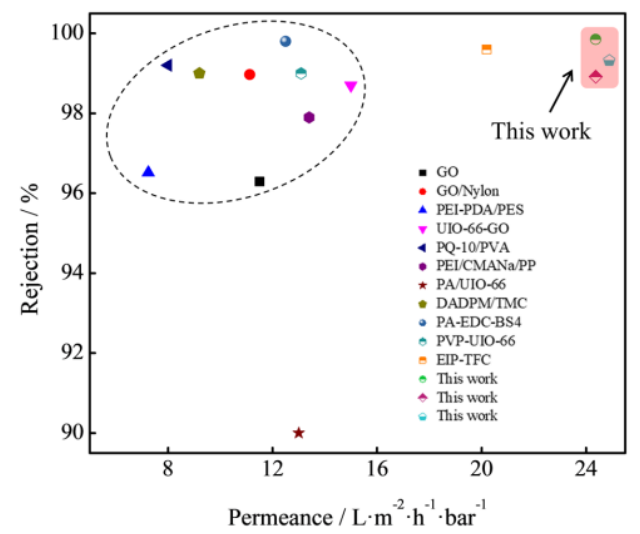

Fig. 12 Nanofiltration performance of the S-EMT/PA-2 membrane compared with other NF membranes (detailed data for the reference membranes are presented in Table 4).

Compared with other reported NF membranes, the S-EMT/PA-2 membrane exhibited enhanced nanofiltration performance (Fig. 12, Table 4). The water permeance of the membrane was about three times higher than that of most other reported membranes and simultaneously had excellent rejection. It can be attributed to the ultra-small EMT zeolite nanocrystals that reduce the generation of the non-selective voids, and lead to the formation of unique ordered pore structure providing additional size sieving effect and improved water molecular transport.

Table 4. Summary of the NF membrane performance from this work (S-EMT/PA-2) and various membranes published.

\begin{tabular}{|c|c|c|c|c|c|}
\hline \multirow{2}{*}{ Membrane } & Dye & $\begin{array}{c}\text { Pressure } \\
(\mathrm{bar})\end{array}$ & $\begin{array}{c}\text { Permeance } \\
\left(\mathrm{L} \mathrm{m}^{-2} \mathrm{~h}^{-1} \mathrm{bar}^{1}\right)\end{array}$ & $\begin{array}{c}\text { Rejection } \\
(\%)\end{array}$ & Ref. \\
\hline \multirow{2}{*}{ S-EMT/PA-2 } & Crystal violet & 1 & 24.37 & $99.98 \%$ & \multirow{2}{*}{ This } \\
\cline { 2 - 5 } & Methylene blue & 1 & 24.37 & $98.92 \%$ & work \\
\cline { 2 - 5 } & Congo red & 1 & 24.48 & $99.32 \%$ & \\
\hline GO & Methylene blue & 1 & 11.5 & $96.29 \%$ & {$[35]$} \\
\hline PEI-PDA/PES & Methylene blue & 1 & 11.13 & $98.97 \%$ & {$[36]$} \\
\hline UIO-66-GO & Methylene blue & 2 & 7.25 & $96.52 \%$ & {$[37]$} \\
\hline PQ-10/PVA & Crystal violet & 7 & 15.0 & $98.7 \%$ & {$[38]$} \\
\hline PEI/CMCNa/PP & Crystal violet & 3 & 13.4 & $97.9 \%$ & {$[40]$} \\
\hline
\end{tabular}




\begin{tabular}{|c|c|c|c|c|c|}
\hline PA/UIO-66 & Crystal violet & 5 & 13.0 & $90 \%$ & {$[41]$} \\
\hline DADPM/TMC & Congo red & 4 & 9.20 & $99 \%$ & {$[42]$} \\
\hline PA-EDC-BS4 & Congo red & 5 & 12.5 & $99.8 \%$ & {$[43]$} \\
\hline PVP-UIO-66 & Congo red & 4 & 13.09 & $99 \%$ & {$[44]$} \\
\hline EIP-TFC & Congo red & 5 & 20.2 & $99.6 \%$ & {$[45]$} \\
\hline
\end{tabular}

\section{Conclusion}

In summary, the spraying method was applied to homogeneously disperse the EMT zeolite nanocrystals during the interfacial polymerization preparation of EMT /PA TFN membranes. The ultra-small EMT zeolite nanocrystals with a size of $\sim 15 \mathrm{~nm}$ were synthesized free of organic template and used as nanofillers. Benefiting from the ultra-small size and the spray dispersion method, the embedded nanocrystals were tightly anchored and uniformly distributed in the PA active layer. Due to the suitable pore structure of EMT zeolite and the excellent compatibility with the polymer, the TFN membrane exhibited high water permeance up to $24.37 \mathrm{~L} \mathrm{~m}^{-2} \mathrm{~h}^{-1} \mathrm{bar}^{-1}$ which was substantially increased compared to the PA TFC membrane; an ideal dye rejection of $99.98 \%$ was measured for the EMT/PA TFN membranes. The results suggest that the incorporation of ultra-small zeolite crystals into the PA layer by spray dispersion method could be a promising way for producing TFN membranes for efficient dye nanofiltration.

\section{Acknowledgements}

This work was supported from Thousand Talents Program for Foreign Experts (WQ20152100284), National Natural Science Foundation of China (Grants No. U1862118, 21501198, 21601205 and 21771193), Taishan Scholar Foundation (ts201511019) and the Fundamental Research Funds for the Central Universities (18CX02047A, 18CX07001A, 18CX05018A, 20CX05010A), PetroChina Innovation Foundation (2019D-5007-0411), Key Research and Development Projects of Shandong Province (2019JZZY010331).

\section{References}

[1] M.C.M. van Loosdrecht, D. Brdjanovic, Anticipating the next century of wastewater treatment, Science, 344 (2014) 1452-1453.

[2] A.K. An, J. Guo, S. Jeong, E.-J. Lee, S.A.A. Tabatabai, T. Leiknes, High flux and 
antifouling properties of negatively charged membrane for dyeing wastewater treatment by membrane distillation, Water Res., 103 (2016) 362-371.

[3] C.-Z. Liang, S.-P. Sun, F.-Y. Li, Y.-K. Ong, T.-S. Chung, Treatment of highly concentrated wastewater containing multiple synthetic dyes by a combined process of coagulation/flocculation and nanofiltration, J. Membr. Sci., 469 (2014) 306-315.

[4] A.K. Verma, R.R. Dash, P. Bhunia, A review on chemical coagulation/flocculation technologies for removal of colour from textile wastewaters, J. Environ. Manage., 93 (2012) 154-168.

[5] M.S. Diallo, Water treatment by dendrimer-enhanced filtration, Nanotechnol. Appl. Clean Water, (2009) 143-155.

[6] G. Zeng, Y. He, Y. Zhan, L. Zhang, Y. Pan, C. Zhang, Z. Yu, Novel polyvinylidene fluoride nanofiltration membrane blended with functionalized halloysite nanotubes for dye and heavy metal ions removal, J. Hazard. Mater., 317 (2016) 60-72.

[7] C. Liu, L. Cheng, Y. Zhao, L. Zhu, Interfacially crosslinked composite porous membranes for ultrafast removal of anionic dyes from water through permeating adsorption, J. Hazard. Mater., 337 (2017) 217.

[8] Mei, Jiang, Kunfeng, Ye, Jiajie, Deng, Jiuyang, Lin, Wenyuan, Shuaifei, Conventional ultrafiltration as effective strategy for dye/salt fractionation in textile wastewater treatment, Environ. Sci. Technol., 52 (2018) 10698-10708.

[9] X. Chen, Y. Zhao, J. Moutinho, J. Shao, A.L. Zydney, Y. He, Recovery of small dye molecules from aqueous solutions using charged ultrafiltration membranes, J. Hazard. Mater., 284 (2015) 58-64.

[10] L. Yu, J. Deng, H. Wang, J.D. Liu, Y. Zhang, Improved Salts Transportation of a Positively Charged Loose Nanofiltration Membrane by Introduction of Poly(ionic liquid) Functionalized Hydrotalcite Nanosheets, ACS Sustain. Chem. Eng., 4 (2016) 3292-3304.

[11] W.J. Lau, A.F. Ismail, Polymeric nanofiltration membranes for textile dye wastewater treatment: preparation, performance evaluation, transport modelling, and fouling control - a review, Desalination, 245 (2009) 321-348.

[12] R. Zhang, S. Ji, N. Wang, L. Wang, G. Zhang and J.-R. Li, Coordination-Driven In Situ Self-Assembly Strategy for the Preparation of Metal-Organic Framework Hybrid Membranes, Angew. Chem., Int. Ed., 53 (2014) , 9775-9779.

[13] L. Wang, S. Ji, N. Wang, R. Zhang, J.R. Li, One-step self-assembly fabrication of 
amphiphilic hyperbranched polymer composite membrane from aqueous emulsion for dye desalination, J. Membr. Sci., 452 (2014) 143-151.

[14] E.M.V. Hoek, V.V. Tarabara, Encyclopedia of Membrane Science and Technology || Nanofiltration, (2013).

[15] C.K. Diawara, Nanofiltration Process Efficiency in Water Desalination, Sep. Purif. Rev., 37 (2008) 302-324.

[16] L.Y. Ng, A.W. Mohammad, C.P. Leo, N. Hilal, Polymeric membranes incorporated with metal/metal oxide nanoparticles: A comprehensive review, Desalination, 308 (2013) 15-33.

[17] J. Yin, Y. Yang, Z. Hu, B. Deng, Attachment of silver nanoparticles (AgNPs) onto thin-film composite (TFC) membranes through covalent bonding to reduce membrane biofouling, J. Membr. Sci., 441 (2013) 73-82.

[18] C. Li, S. Li, L. Tian, J. Zhang, B. Su, M.Z. Hu, Covalent organic frameworks (COFs)-incorporated thin film nanocomposite (TFN) membranes for high-flux organic solvent nanofiltration (OSN), J. Membr. Sci., 572 (2019) 520-531.

[19] Tae, Hoon, Lee, Jee, Yeon, Oh, Sung, Pyo, Hong, ZIF-8 particle size effects on reverse osmosis performance of polyamide thin-film nanocomposite membranes: Importance of particle deposition, J. Membr. Sci., 15 (2019) 23-33.

[20] B.H. Jeong, E.M.V. Hoek, Y. Yan, A. Subramani, X. Huang, G. Hurwitz, A.K. Ghosh, A. Jawor, Interfacial polymerization of thin film nanocomposites: A new concept for reverse osmosis membranes, J. Membr. Sci., 294 (2007) 1-7.

[21] J. Zhu, L. Qin, A. Uliana, J. Hou, J. Wang, Y. Zhang, X. Li, S. Yuan, J. Li, M. Tian, Elevated Performance of Thin Film Nanocomposite Membranes Enabled by Modified Hydrophilic MOFs for Nanofiltration, ACS Appl. Mater. Interfaces., 9 (2017) 1975-1986.

[22] N. Ghaemi, P. Safari, Nano-porous SAPO-34 enhanced thin-film nanocomposite polymeric membrane: Simultaneously high water permeation and complete removal of cationic/anionic dyes from water, Journal of Hazardous Materials, 358 (2018) 376-388. [23] H. Fan, N. Wang, S. Ji, H. Yan, G. Zhang, Nanodisperse ZIF-8/PDMS hybrid membranes for biobutanol permselective pervaporation, J. Mater. Chem. A, 2 (2014) 20947-20957.

[24] Z. Chen, B. Holmberg, W. Li, X. Wang, W. Deng, R. Munoz, Yan, Nafion/Zeolite Nanocomposite Membrane by in Situ Crystallization for a Direct Methanol Fuel Cell, 
Chem. Mater., 18 (2006) 5669-5675.

[25] A.M.A. Abdelsamad, A.S.G. Khalil, M. Ulbricht, Influence of controlled functionalization of mesoporous silica nanoparticles as tailored fillers for thin-film nanocomposite membranes on desalination performance, J. Membr. Sci., 563 (2018) 149-161.

[26] E.P. Ng, D. Chateigner, T. Bein, V. Valtchev, S. Mintova, Capturing Ultrasmall EMT Zeolite from Template-Free Systems, Science, 335 (2012) 70-73.

[27] V. Georgieva, A. Vicente, C. Fernandez, R. Retoux, A. Palcic, V. Valtchev, S. Mintova, Control of Na-EMT Zeolite Synthesis by Organic Additives, Cryst. Growth Des., 15 (1906) 1898-1906.

[28] U. Thubsuang, H. Ishida, S. Wongkasemjit, T. Chaisuwan, Novel template confinement derived from polybenzoxazine-based carbon xerogels for synthesis of ZSM-5 nanoparticles via microwave irradiation, Microporous Mesoporous Mater., 156 (2012) 7-15.

[29] M.F. Jimenez Solomon, Y. Bhole, A.G. Livingston, High flux membranes for organic solvent nanofiltration (OSN)-Interfacial polymerization with solvent activation, J. Membr. Sci., 423 (2012) 371-382.

[30] A.K. Ghosh, E.M.V. Hoek, Impacts of support membrane structure and chemistry on polyamide-polysulfone interfacial composite membranes, J. Membr. Sci., 336 (2009) 140-148.

[31] R. N. Resistance of solid surfaces to wetting by water, Ind. Eng. Chem., 28 (1936), 988-994.

[32] J. Palmeri, J. Sandeaux, R. Sandeaux, X. Lefebvre, P. David, C. Guizard, P. Amblard, J.F. Diaz, B. Lamaze, Modeling of multi-electrolyte transport in charged ceramic and organic nanofilters using the computer simulation program NanoFlux, Desalination, 147 (2002) 231-236.

[33] C. Mazzoni, F. Orlandini, S. Bandini, Role of electrolyte type on $\mathrm{TiO}_{2}-\mathrm{ZrO}_{2}$ nanofiltration membranes performances, Desalination, 240 (2009) 227-235.

[34] L. Huang, J. Chen, T. Gao, M. Zhang, Y. Li, L. Dai, L. Qu, G. Shi, Reduced Graphene Oxide Membranes for Ultrafast Organic Solvent Nanofiltration, Adv. Mater., 28 (2016) 8669-8674. 
[35] L. Chen, Y. Li, L. Chen, N. Li, C. Dong, Q. Chen, B. Liu, Q. Ai, P. Si, J. Feng, A large-area free-standing graphene oxide multilayer membrane with high stability for nanofiltration applications, Chem. Eng. J., (2018) 536-544.

[36] Chen, Long, Moon, Jung-Hyeon, Ma, Xiaoxin, Zhang, Lin, Qiong, Lina, High performance graphene oxide nanofiltration membrane prepared by electrospraying for wastewater purification, Carbon, 130 (2018) 487-494.

[37] R. Zhang, Y. Su, X. Zhao, Y. Li, J. Zhao, Z. Jiang, A novel positively charged composite nanofiltration membrane prepared by bio-inspired adhesion of polydopamine and surface grafting of poly(ethylene imine), J. Membr. Sci., 470 (2014) $9-17$.

[38] K. Guan, D. Zhao, M. Zhang, J. Shen, W. Jin, 3D nanoporous crystals enabled $2 \mathrm{D}$ channels in graphene membrane with enhanced water purification performance, $\mathrm{J}$. Membr. Sci., 542 (2017) 41-51.

[39] Y. Zheng, G. Yao, Q. Cheng, S. Yu, C. Gao, Positively charged thin-film composite hollow fiber nanofiltration membrane for the removal of cationic dyes through submerged filtration, Desalination, 328 (2013) 42-50.

[40] Q. Chen, P. Yu, W. Huang, S. Yu, M. Liu, C. Gao, High-flux composite hollow fiber nanofiltration membranes fabricated through layer-by-layer deposition of oppositely charged crosslinked polyelectrolytes for dye removal, J. Membr. Sci., 492 (2015) 312-321.

[41] X. Cheng, X. Jiang, Y. Zhang, C.H. Lau, Z. Xie, D. Ng, S.J.D. Smith, M.R. Hill, L. Shao, Building Additional Passageways in Polyamide Membranes with Hydrostable Metal Organic Frameworks to Recycle and Remove Organic Solutes from Various Solvents, ACS Appl. Mater. Interfaces., 9 (2017) 38877-38886.

[42] C. Yang, W. Xu, Y. Nan, Y. Wang, X. Chen, Novel negatively charged nanofiltration membrane based on 4,4'-diaminodiphenylmethane for dye removal, Sep. Purif. Technol., 248 (2020) 117089.

[43] M.L. A, Q.H. B, K.Z. B, Z.G. B, Z.L. b, S.Y. B, C.G. C, Carbodiimide-assisted zwitterionic modification of poly(piperazine amide) thin-film composite membrane 
for enhanced separation and anti-depositing performances to cationic/anionic dye aqueous solutions, J. Hazard. Mater., 396 (2020) 122582 .

[44] P. Zhao, R. Li, W. Wu, J. Wang, J. Liu, Y. Zhang, In-situ growth of polyvinylpyrrolidone modified Zr-MOFs thin-film nanocomposite (TFN) for efficient dyes removal, Composites, 176 (2019)107201-107208.

[45] Y. Kang, J. Jang, S. Kim, J. Lim, I.S. Kim, PIP/TMC Interfacial Polymerization with Electrospray: Novel Loose Nanofiltration Membrane for Dye Wastewater Treatment, ACS Appl. Mater. Interfaces, 12 (2020) 36148-36158. 